\title{
Research on Multi-disciplinary Collaborative Simulation Based on Interfaces Used in Tracked Vehicle
}

\author{
LI San-qun ${ }^{1, ~ a, ~ H U ~ Z h o n g-l i n g ~}{ }^{1, ~ a}$, JIA Chang-zhi ${ }^{1, ~ a ~}$, HE Yao-xin ${ }^{1, \text { a }}$ \\ ${ }^{1}$ Department of Guns Engineering, Ordnance Engineering College, Shijiazhuang, 050003 \\ a E-mail: hzloec@163.com
}

Keywords: tracked vehicle, interfaces, collaborative simulation, planetary framework

\begin{abstract}
Due to the limit of the static strength theory design used in tracked vehicle, it can't reflect the dynamic loads suffered by the main part, so it is difficult to analyze the reliability and remained lifetime prediction, in some degree it affect the performance. In this paper, we apply the collaborative simulation based on interfaces to tracked vehicle, build the flow chart, do the research on lifetime prediction and structure optimization of tracked vehicle based on driving simulation test, and then prove its feasibility with an example of planetary framework. The research on collaborative simulation based on interfaces used in tracked vehicle not only solves the technical problem which caused by complex task profile, but also joins the engineer tightly, it is significant in practice.
\end{abstract}

\section{Introduction}

Currently tracked vehicle plays an important role in the modern military, agriculture, construction and other fields by virtue of its good pass performance. The research on tracked vehicle's performance has been remained on the basis of "experience + test" all the time, on the need to establish a large number of empirical formulas, make statistics of a large number of experimental data, study during a long period, especially for the many factors that affect the performance of tracked vehicle, it is difficult to have quantitative identification and characterization. According to a survey, due to the lack of accurate load data, the design of a type of tracked vehicle calculated the intensity of the working load by considering the weight act on driving wheel, and the maximum possible load, in some extreme conditions, such as braking, skidding and crossing a single obstacle on the pavement, according to the accumulated experience and information to design, safety factor of components designed generally got too large. Although the working reliability increased in this way, the load tracked vehicle parts withstand is largely alternating dynamic load which is caused by the degree of uneven load and the entire vehicle's vibration, its size and characteristic are affected by road conditions, using conditions, structural parameters of the entire system and its components and many other factors, thus resulting in tracked vehicle's not high reliability. By the experience of using automotive, it shows that nearly $70 \%$ of the parts are damaged not under the maximum load but under dynamic load in the process due to fatigue. Therefore, the determination of the real dynamic load which the components designed to withstand is a basic and important work.

Currently, the stochastic dynamic loads of driving system are mostly determined by road test, the resulting data is certainly reliable in theory, nonetheless it should be seen that the using conditions of vehicle are extremely complex, loads change largely, some road tests on the typical road cannot produce a reasonable description of the whole driving conditions, the method of measuring the loads of all the driving conditions causes the long test period, the heavy data processing, the large cost, the slow effect, and for the limit from test means, some tests are unable to be done.

With the development of multi-body dynamics, computer software and hardware, and the emergence of virtual prototype technology, complex mechanical system simulation proves to be possible and researching on reliability analysis and lifetime prediction of different parts of the task profile case to withstand dynamic loads proves to be possible. This paper applied collaborative simulation technology based on interfaces to tracked vehicle, aimed at the phenomenon of poor 
reliability of key pieces of weaponry systems and consumables, to do failure mechanism analysis, structural optimization designing and lifetime prediction and other multi-disciplinary crossing research, to provide reliable reference data for the entire vehicle, for the performance of the tracked vehicle be fully played.

\section{Multi-disciplinary modeling and simulation}

Mainly due to the research and application in the military field, computer simulation got a large development, and then it gradually applied to engineering fields. In the military field, the United States, based on successful experiences, regards the "Synthetic Simulation Environment" as one of the seven areas of science and technology to promote the development of national defense science and technology. As technology advances, the multi-body dynamics, collision, aerodynamics and structural analysis and other types of simulation, has been widely used in the design of mechanical products. Through the simulation of various properties of the product (such as car's crashing characteristic, aerodynamic characteristic, operability, fatigue resistance characteristic), the mechanical design can be verified and optimized. Computer simulation technology is a typical representative of this trend, and as computer technology continues to mature, it will become a hard-striking and danger-attacking weapon. Germany's BMW Company uses computer simulation technology, reduces a lot of money consumed by the physical model, and thus ensuring its competitive advantage. Boeing 777 aircraft used the computer simulation technology acquired the success of design and production without drawing, that was a major breakthrough caused the scientific community, the business community's attention in recent years, greatly reduced the original seven to eight years or even longer design cycle, achieved the successfully first test flight from design to target during three years.

Multi-disciplinary modeling is to "assembly" the models derived from mechanics, control, electronics, hydraulic pressure, pneumodynamics, software and a number of models in different disciplines into a larger simulation model for emulation. When the multi-disciplinary modeling is completed, the model in different disciplines desire mutual coordination and working together to complete the simulation running, that is multi-disciplinary collaborative simulation running.

At present, there are mainly two approaches in multi-disciplinary modeling, one is the interfaces based approach; the other is the uniform language based approach. Interfaces-based approach, gives full play to all areas of commercial simulation software's specialities in their respective fields and uses them to complete simulation model building in their respective fields, and bases on the interfaces between the various areas of commercial simulation software to achieve multi-disciplinary modeling and simulation. After the use of multi-disciplinary modeling based on interfaces has completed complex products' multi-disciplinary collaborative simulation modeling, it usually requires the use of all areas of commercial simulation software's collaborative simulation running function based on interfaces to achieve different areas of commercial simulation software modeling, get different simulation running among different models. Uniform language-based approach, is to use one uniform language, such as BOND diagram, to achieve multi-disciplinary modeling. However, due to the development of uniform language-based multi-disciplinary modeling approach is later than the multi-disciplinary modeling interfaces-based approach, it mainly confined research field, with few actual use. Currently interfaces-based approach is the most frequently used approach, which is firstly use one area of commercial simulation software to complete simulation model's building in its respective fields, and use the interfaces between the various areas of commercial simulation software to achieve multi-disciplinary modeling and simulation.

In this paper, as an advanced research tool, the multi-disciplinary modeling and simulation based on interfaces was introduced into the study in tracked vehicle system, during the process of development and use of tracked vehicle, simulation technology was used to replace physical prototype with virtual prototype to test, so that the new technology, new concepts, new programs repeated test, analysis and comparison in a virtual environment, used knowledge from people's 
accumulation in the field of cross-disciplinary, to determine the best option in order to select an optimal technical approach.

\section{Research on collaborative simulation based on interfaces used in tracked vehicle}

To apply multi-disciplinary co-simulation based on interfaces to track vehicles, in which an foundational and important aspect is closely linked engineering practical application, collection and analysis of tracked vehicle reliability data, on this basis, with regard to the problems occurs in tracked vehicle, by simulation, suit the remedy to the case, to determine the system's weaknesses, once the weak link in the overall system reliability is determined, it is feasible to use collaborative modeling and simulation analysis of multi-disciplinary field to do kinetic analysis, lifetime prediction and structure optimization of the system's weaknesses, thereby improving the reliability of the tracked vehicle. Figure 1 shows the flow chart about multi-disciplinary co-simulation based on interfaces applied to the study of tracked vehicle.

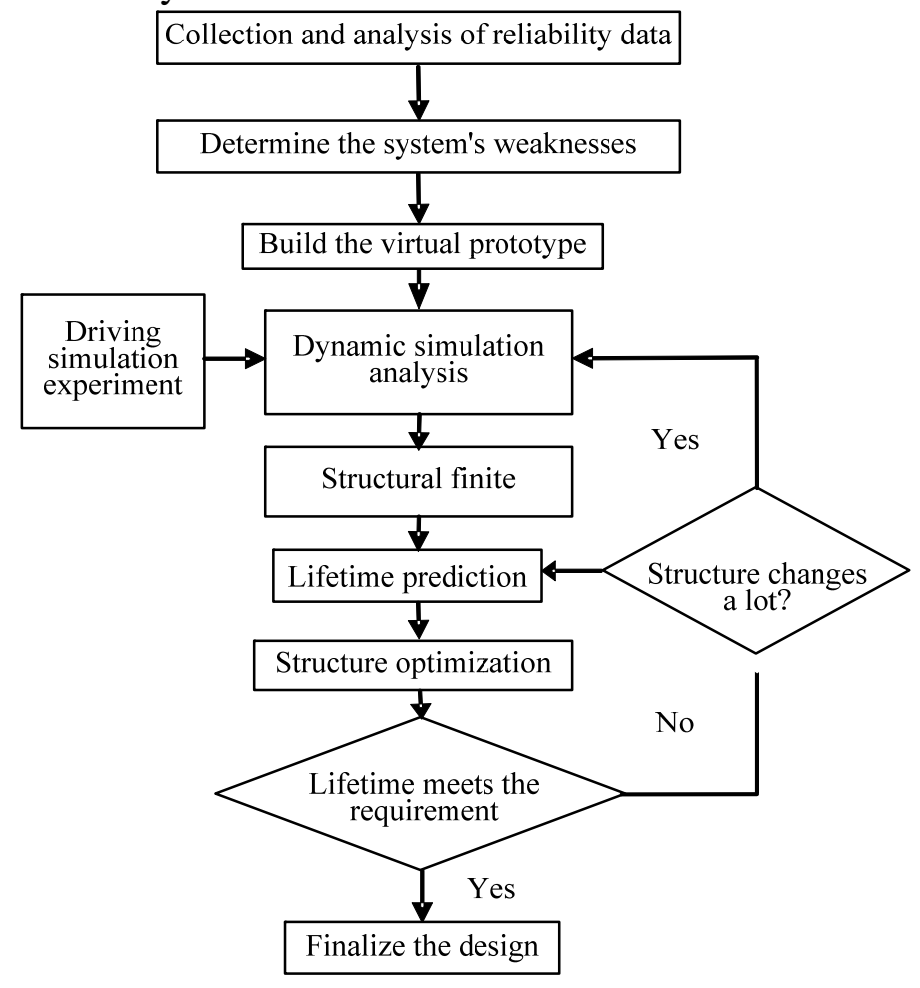

Figure 1 The flow chart about multi-disciplinary co-simulation based on interfaces applied to the study of tracked vehicle.

As can be seen from the flow chart of Figure 1, the flow chart involved multi-disciplinary field simulation software, such as mechanical multi-body dynamics simulation software, fatigue lifetime analysis and simulation software, mechanical structural finite element analysis software and other software, and the most important, on the basis of driving simulation test, we obtained the dynamic load that tracked vehicle withstand in different task profile, solved the technical problems encountered when analyzed tracked vehicle. With the interfaces during different disciplines of commercial software, the multi-disciplinary co-simulation model of lifetime prediction and structural optimization of tracked vehicle can be obtained, and running multi-disciplinary collaborative simulation, completing the components' lifetime prediction and structural optimization analysis under dynamic load can be achieved. As shown in Figure 2, these simulation models in different areas of discrete time points in the simulation, achieve the mutual exchange of information via interprocess communication (IPC) and other methods, then use their solver (also known as integrator) to solve, in order to complete the entire system's simulation of lifetime prediction and structure optimization under dynamic load. 


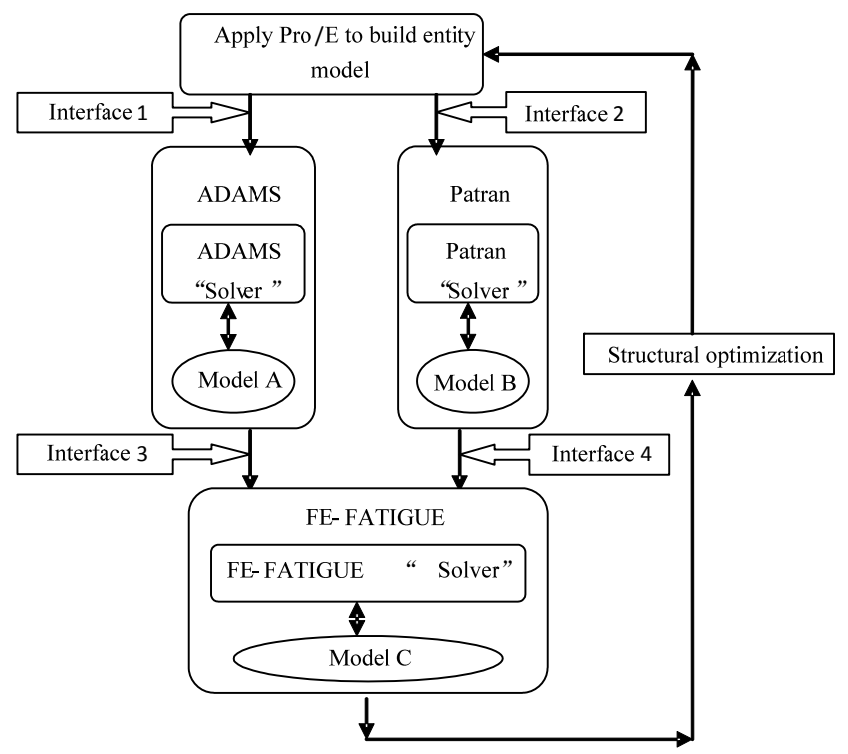

Figure 2 The coordination of the application of multi-disciplinary collaborative simulation technology based on interfaces to tracked vehicle.

\section{Research on multi-disciplinary collaborative simulation of planetary framework}

In order to verify the feasibility of the multi-disciplinary collaborative simulation based on interfaces applying to tracked vehicle, as an example, this paper based on the planetary gears in tracked vehicle, according to the flow chart provided in Figure 1, with the collaborative relationship among different simulation fields, to achieve interdisciplinary, built the virtual prototype (model A) of planetary gears in tracked vehicle based on tracked vehicles' driving simulation test, and did the dynamic simulation analysis, thus providing the dynamic load of the planetary gears studied in this paper in different task profiles runtime. From the collection and analysis of reliability data, the fault frequency of planetary framework in planetary transmission appeared in the application to tracked vehicle is higher than others, the high fault level of planetary framework is the most important thing, seriously affected the reliability of tracked vehicle, so put the planetary framework as one of the weak links in tracked vehicle, completed the multi-disciplinary collaborative simulation under dynamic load, to provide reliable data base for the planetary framework lifetime prediction and structure optimization to improve its overall reliability.

As the simulation coordination among various business software applied in this paper shown in Figure 2, the driving simulation test provided the dynamic load that the dangerous section of the planet bore, used multi-body dynamics analysis software ADAMS to establish the virtual prototype model A of the planetary gear, and with the combination of mechanical structural finite element analysis software MSC.Patran, did structural mechanics analysis of planetary framework and built model B, used the interface between MSC.Patran and FE-FATIGUE to make fatigue lifetime prediction and built model $\mathrm{C}$, integration and debugging were done on the results obtained from the single-disciplinary simulation software, so as to achieve the purposes to do mechanics analysis, lifetime prediction and structure optimization of mechanical parts. The research results carried out from the planetary framework was shown in Figure 3. 


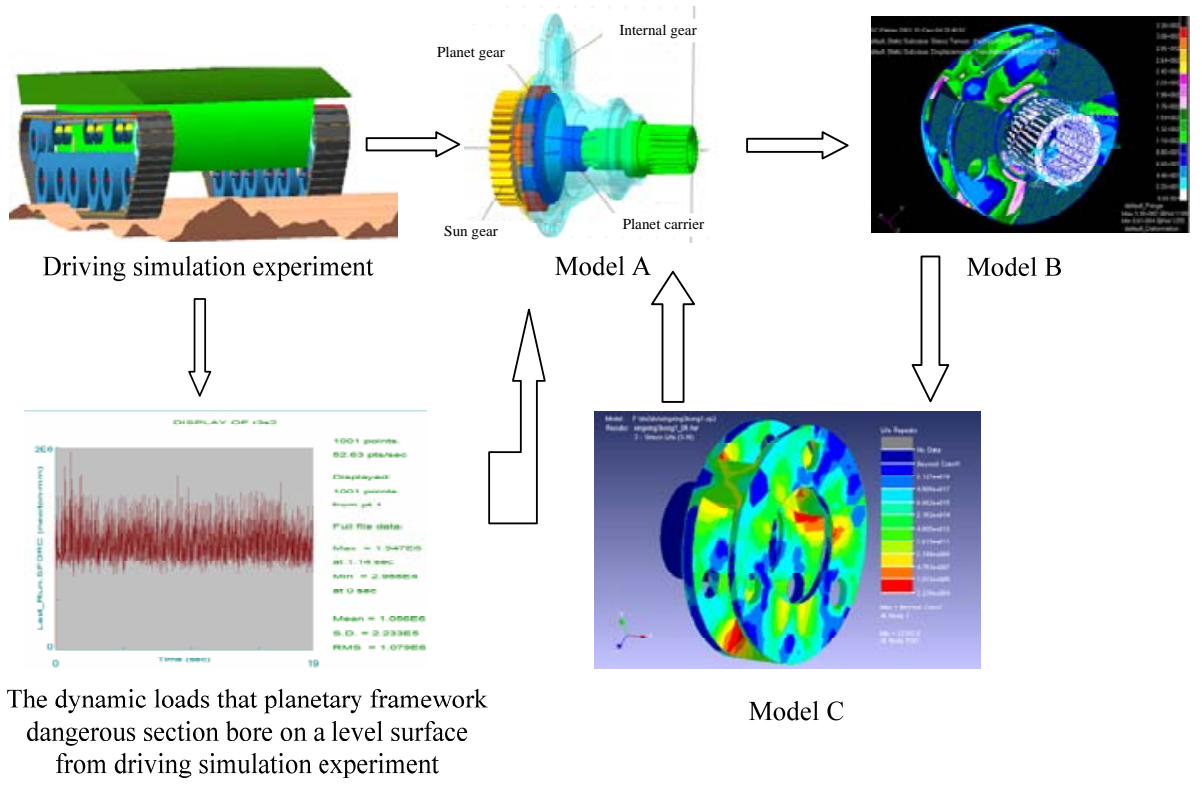

Figure 3 An available example of the application of multi-disciplinary collaborative simulation based on interfaces to tracked vehicle.

By as shown in Figure 3, model A was the virtual prototype model of tracked vehicle planetary transmission; Model B was the finite element analysis of framework (planetary framework was made of three planetary gear transmission structures); Model $\mathrm{C}$ was the fatigue lifetime prediction of planet framework. Knowing from the available example of the application to the planet framework, on the basis of driving simulation test of tracked vehicle, the lifetime prediction of planetary framework of planetary transmission device could be done with the use of multi-disciplinary simulation based on interfaces, for the next step to carry on dynamic structure optimization and residual lifetime prediction of the planetary framework to provide important reference basis.

\section{Conclusions}

This paper aimed at a technical problem which is the large gap between design lifetime and actual lifetime of tracked vehicle, due to the congenital deficiency of static strength design theory, reliability and lifetime of tracked vehicle under the influence of dynamic loads that tracked vehicle components suffered under an actual task profile cannot be reflected by parts. This paper took advantage of the organic combination of multi-disciplinary co-simulation technology based on interfaces and tracked vehicle, on an simulation experimental basis, to provide the dynamic load that tracked vehicle suffered, predict the lifetime under the influence of dynamic loads from different task profile circumstances, and use an actual example to demonstrate the feasibility of this approach in tracked vehicle. Multi-disciplinary collaborative simulation based on interfaces' application to tracked vehicle has a very important practical value, with a very wide range of application prospect, it will produce great economic benefits.

\section{REFERENCES}

[1] Jilin University of Technology, Auto Teaching and Research Section. Car Design. Beijing: China Machine Press, 1983.

[2] HE Guo-fang, XU Hai-bao. Beijing Collection and Analysis of Reliability Data.: National Defense Industry Press, 1995, 12, Version 1. 
[3] XIONG Guang-leng, GUO Bin. Collaborative Technology and Virtual Prototype Technology. Beijing: Tsinghua University Press, 2003, 8, Version 1.

[4] CHEN Xiao-bo, XIONG Guang-leng. Research on Collaborative Simulation Operation Based on HLA. Journal of System Simulation, 2003, 15(12): 1707-1712.

[5] DU Xiu-ju, DONG Zhao-wei, Etc. Research on Collaborative Simulation Based on Interface Used in Weapon System. Journal of System Simulation, 2006, 5: 1371-1374.

[6] D. Rubinstein, R. Hitron. A detailed multi-body model for dynamic simulation of off-road tracked vehicles, Journal of Terramechanics 41 (2004) 163-173.

[7] J. Yamakawa, K. Watanabe. A spatial motion analysis model of tracked vehicles with torsion bar type suspension. Journal of Terramechanics 41 (2004) 113-126.

[8] 